\title{
Precursor nanoscale modulations in ferromagnets: Modeling and thermodynamic characterization
}

\author{
Marcel Porta, Teresa Castán, and Antoni Planes \\ Departament d'Estructura i Constituents de la Matèria, Universitat de Barcelona, Diagonal 647, 08028 Barcelona, Catalonia, Spain \\ Avadh Saxena \\ Theoretical Division, Los Alamos National Laboratory, Los Alamos, New Mexico 87545, USA \\ (Received 23 March 2005; revised manuscript received 16 June 2005; published 5 August 2005)
}

\begin{abstract}
Using a Ginzburg-Landau model for the magnetic degrees of freedom with coupling to disorder, we demonstrate through simulations the existence of stripelike magnetic precursors recently observed in $\mathrm{Co}-\mathrm{Ni}-\mathrm{Al}$ alloys above the Curie temperature. We characterize these magnetic modulations by means of the temperature dependence of local magnetization distribution, magnetized volume fraction, and magnetic susceptibility. We also obtain a temperature-disorder strength phase diagram in which a magnetic tweed phase exists in a small region between the paramagnetic and dipolar phases.
\end{abstract}

DOI: 10.1103/PhysRevB.72.054111 PACS number(s): 75.30.Kz, 75.40.Mg, 61.43.-j, 81.30.Kf

\section{INTRODUCTION}

In many martensitic materials ${ }^{1}$ and shape memory alloys ${ }^{2}$ it is well established through high-resolution transmission electron microscopy (TEM) that cross-hatched nanoscale strain modulations exist above the structural transition temperature. This type of precursors is known as (structural) tweed. ${ }^{3}$ The tweed has been suitably characterized ${ }^{4}$ through inelastic neutron diffuse scattering, ${ }^{5}$ anisotropic thermal expansion, ${ }^{6}$ phonon anomalies, ${ }^{7}$ etc. Its origin is attributed to compositional fluctuations in these alloys coupling to the transformation strain. Simulations of a model that embodies these notions clearly reproduce the observed tweed microstructure and the associated diffuse scattering. ${ }^{8-10}$

From both experiments and theory it is now well understood that cross-hatched anisotropic correlations observed by TEM are indicative of the sensitivity of long-range (elastic) strain response to local perturbations. ${ }^{11}$ Then, a natural question arises: is this phenomenon specific to certain structural transformations, in particular alloys, or is it a much more general concept applicable to other ferroic properties such as polarization in ferroelectrics or magnetization in magnetic alloys? The answer to the latter is in the affirmative: Recently tweedlike precursors have been observed not only in ferroelectrics ${ }^{12-14}$ and magnetic alloys above the Curie temperature $^{15}$ but also in magnetoelastic ${ }^{16-19}$ materials. Clearly, the tweed concept is much more general in a wide variety of materials than previously thought. Indeed, it is a clear example of the sensitivity of materials properties to perturbations arising from intrinsic inhomogeneities of the system. ${ }^{20}$

Magnetic tweed has been observed in $\mathrm{Co}_{0.38} \mathrm{Ni}_{0.33} \mathrm{Al}_{0.29}$ alloys. ${ }^{15}$ Modulations in the magnetization above the Curie point $\left(T_{c}\right)$ with stripelike correlations have been revealed by electron holography ${ }^{21}$ and Lorentz microscopy. ${ }^{22}$ On the other hand, a Ginzburg-Landau model that only involves magnetic degrees of freedom has been recently introduced. ${ }^{23}$ This is a mesoscopic model that invokes magnetic (quenched-in) disorder and long-range magnetic dipolar interactions. The statistical disorder locally modulates the $\mathrm{Cu}$ - rie temperature whereas the long-range dipolar forces produce a global response in terms of a stripelike pattern above $T_{c}$. This is precisely the magnetic tweed with stripes being either vertical or horizontal due to magnetic anisotropy. We note that similar approaches have been used to study inhomogeneous patterns ${ }^{24}$ in more complex transition metal functional perovskites such as colossal magnetoresistance (CMR) manganites and high-temperature superconducting (HTS) cuprates.

Here we explicitly solve the model by means of numerical simulations and demonstrate unambiguously the existence of such precursor nanoscale modulations in the paramagnetic phase. A more important issue is the thermodynamic characterization of tweed. This is achieved by calculating the temperature dependence of different physical quantities such as the local magnetization distribution, the magnetized volume fraction, and the inverse magnetic susceptibility. From this last quantity, the tweed region is identified as that giving rise to deviation from the linear Curie law behavior within the paramagnetic phase. Consistently, the local magnetization distribution shows a single peak centered at zero magnetization, with a finite width that increases from zero when cooling within the paramagnetic phase, indicating the onset of tweed. Upon further cooling, the peak gets broader and turns into a two peak symmetrical distribution in the dipolar (ferromagnetic) phase. Additionally, the volume fraction of magnetic domains increases gradually from zero at the onset of tweed and exhibits an inflection point at the Curie temperature. This behavior seems to indicate a crossover from the paramagnetic phase into the tweed phase. The tweed is separated from the low-temperature dipolar phase by an essentially continuous transition (at $T_{c}$ ), consistent with experimental observations. ${ }^{15}$ Furthermore, we have explored the tweed stability region for different values of the strength of disorder and constructed a phase diagram. For completeness, we have also calculated the size distribution of magnetic domains and find that in the tweed region it exhibits a broad, well defined single peak. This peak abruptly switches to bigger sizes at the transition temperature $T_{c}$. 
The plan of the paper is as follows. In the next section we present our model for a two-dimensional paramagnetic to ferromagnetic transition. For convenience, all calculations involving the dipolar interaction are given in detail in the Appendix. In Sec. III we present relaxational simulations that clearly demonstrate magnetic tweed patterns above the Curie temperature and provide a phase diagram in analogy with the phase diagram for the structural tweed. ${ }^{8}$ Finally, we summarize our main findings in Sec. IV with some comments on magnetoelastic and ferroic tweed.

\section{MODEL}

This section is devoted to a discussion of some relevant aspects of the model ${ }^{23}$ recently introduced within the framework of the Ginzburg-Landau approach to describe tweedlike stripe modulations in magnetic materials. Our level of description focuses on the mesoscopic scale at which magnetic modulations (around $50-100 \mathrm{~nm}$ ) have been observed..$^{15}$ Therefore, the microscopic degrees of freedom have been integrated out (in favor of a mesoscopic magnetization order parameter) and the atomistic behavior remains unspecified. This "coarse graining" renders the approach very general and useful because of its simplicity.

The Ginzburg-Landau free energy for a two-dimensional ferromagnet to paramagnet transition is written as the summation of three contributions

$$
F=F_{L}+F_{\text {grad }}+F_{\text {dip }} .
$$

The first term is the homogeneous Landau free energy, consistent with the symmetries of the underlying lattice, and takes into account the magnetic exchange and crystal field interactions

$$
F_{L}=\int\left\{A\left[T-T_{c}(\mathbf{r})\right] m^{2}(\mathbf{r})+B m^{4}(\mathbf{r})+f_{A}(\mathbf{m})\right\} d \mathbf{r},
$$

where $A$ and $B$ are positive constants and $f_{A}(\mathbf{m})$ denotes the magnetic anisotropy function that we shall discuss later. The coupling of disorder (e.g., compositional disorder in alloys) to magnetism is taken into account through the Curie temperature, which is assumed to fluctuate in space, $T_{c}(\mathbf{r})=T_{c}^{o}$ $+\eta(\mathbf{r})$, where $T_{c}^{o}$ is the nominal value of the Curie temperature in the absence of long-range forces (described below). The disorder is modeled by the random variable $\eta(\mathbf{r})$, which is Gaussian distributed with zero mean and the following pair correlation function

$$
\left\langle\eta(\mathbf{r}) \eta\left(\mathbf{r}^{\prime}\right)\right\rangle=\frac{\varepsilon}{2 \pi \sigma^{2}} \exp \left(\frac{-\left|\mathbf{r}-\mathbf{r}^{\prime}\right|}{\sigma}\right),
$$

where $\sigma$ is the disorder correlation length and $\varepsilon$ is the strength of disorder.

The second term in Eq. (1) is the contribution to the total free energy due to the gradient of the order parameter $\mathbf{m}(\mathbf{r})$, which is the energy cost (proportional to $D$ ) for creating magnetic domain walls. Its expression is written in the usual way:

$$
F_{\text {grad }}=D \int d \mathbf{r} \sum_{i, k=x, y}\left(\frac{\partial m_{i}(\mathbf{r})}{\partial r_{k}}\right)^{2}
$$

In addition to the two short-range contributions discussed above, we have included the long-range dipolar magnetic interaction that enables us to minimize the total magnetostatic energy. For an interaction strength of $G$ in two dimensions it can be written as $^{25}$

$$
\begin{aligned}
F_{\text {dip }}= & \frac{1}{2} G \int d \mathbf{r} \int d \mathbf{r}^{\prime}\left\{\frac{\mathbf{m}(\mathbf{r}) \cdot \mathbf{m}\left(\mathbf{r}^{\prime}\right)}{\left|\mathbf{r}-\mathbf{r}^{\prime}\right|^{2}}\right. \\
& \left.-2 \frac{\left[\mathbf{m}(\mathbf{r}) \cdot\left(\mathbf{r}-\mathbf{r}^{\prime}\right)\right]\left[\mathbf{m}\left(\mathbf{r}^{\prime}\right) \cdot\left(\mathbf{r}-\mathbf{r}^{\prime}\right)\right]}{\left|\mathbf{r}-\mathbf{r}^{\prime}\right|^{4}}\right\} .
\end{aligned}
$$

It is worth noting that although the dipolar energy is nonlocal in real space, it becomes local in Fourier space, ${ }^{23,25}$ provided the size of the system is infinite. Its expression in Fourier space is

$$
\begin{aligned}
F_{\text {dip }}= & 2 \pi^{3} G \int d \mathbf{k} \frac{1}{k^{2}}\left\{\left(k_{x}^{2}-k_{y}^{2}\right)\right. \\
& \times\left[m_{x}(\mathbf{k}) m_{x}(-\mathbf{k})-m_{y}(\mathbf{k}) m_{y}(-\mathbf{k})\right] \\
& \left.+2 k_{x} k_{y}\left[m_{x}(\mathbf{k}) m_{y}(-\mathbf{k})+m_{y}(\mathbf{k}) m_{x}(-\mathbf{k})\right]\right\} .
\end{aligned}
$$

From Eq. (6) it is clear that the dipolar interaction favors modulations of the magnetization in the direction perpendicular to the magnetization vector. For example, for $\mathbf{m}$ $=(0, m)$ the free energy is minimized when the term $\left(k_{x}^{2}-k_{y}^{2}\right) / k^{2}$ is maximum, that is, for $\mathbf{k}=(k, 0)$. Moreover, the value of such a term is exactly the same for all wave vectors parallel to the $x$ direction except for $\mathbf{k}=0$. In this last case, the energy is higher. ${ }^{26}$ This means that for an infinite system the dipolar interaction does not set a domain-wall separation length scale. ${ }^{27}$

In order to perform numerical simulations, the integrals in Eqs. (2), (4), and (5) are replaced by a summation over a discrete two-dimensional lattice. Moreover, the summation is limited to a finite volume. In this way, we take into account finite size and shape anisotropy effects. This is achieved by assuming that the whole system is a finite number of copies of the simulated cell. As it is discussed in the Appendix, under these conditions the dipolar energy depends on the modulation wave vector, and thus, the competition between the dipolar and gradient terms of the free energy stabilizes a characteristic magnetic modulation wavelength. It can be shown that in the low-temperature phase the domain wall separation length $\lambda$ scales with the size of the system $L$ as $^{28}$ $\lambda \sim \sqrt{L}$. The magnetic anisotropy function in Eq. (2) is chosen to be uniaxial in order to avoid competition between different magnetization directions $\left[f_{A}(\mathbf{m})=C m_{x}^{2}(\mathbf{r})\right.$ with $C$ being the crystal field].

We simplify even further and work in the limit of infinite uniaxial anisotropy and focus on the study of one kind of stripes only. This turns out to be very convenient for computing purposes and leaves unmodified our main conclusion concerning the origin and characterization of magnetic tweed. ${ }^{29}$ The model parameters $A, T_{c}^{o}$, and $D$ are set ${ }^{30}$ to unity in order to define the units of length, temperature, and en- 


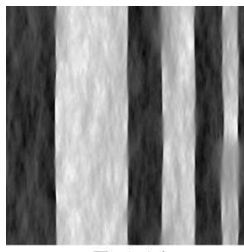

$\mathrm{T}=1.14$

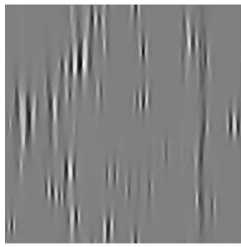

$\mathrm{T}=1.165$

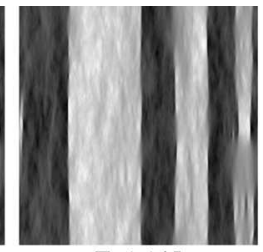

$\mathrm{T}=1.145$

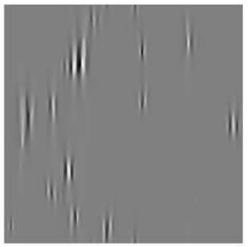

$\mathrm{T}=1.17$

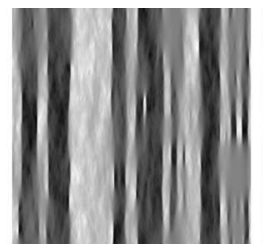

$\mathrm{T}=1.15$

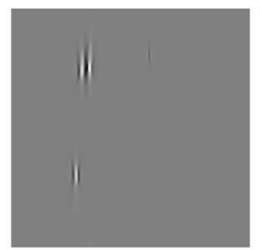

$\mathrm{T}=1.175$

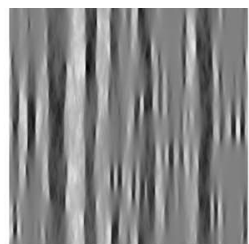

$\mathrm{T}=1.155$

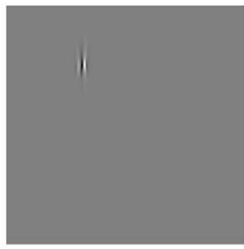

$\mathrm{T}=1.18$

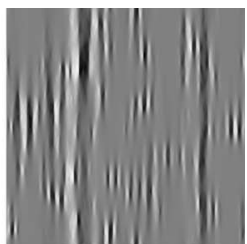

$\mathrm{T}=1.16$

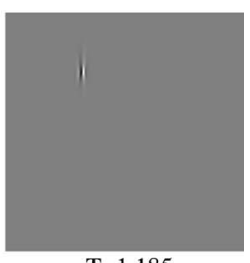

$\mathrm{T}=1.185$
FIG. 1. Snapshots of stable magnetic structures at different temperatures. White (black) regions are up (down) magnetized regions and gray represents nonmagnetized areas. The system size is $L_{x}=L_{y}=512$ and $n_{x}=4, n_{y}=32$. ergy, whereas the parameter $B$ is set to $1 / 2$ in order to define the unit of magnetization. We therefore use reduced units. The remaining parameters are the strength of the dipolar interaction $G$, the strength of disorder $\varepsilon$, and its corresponding correlation length $\sigma$. The size and shape of the system depend on the number of replicas of the simulation cell $n_{x(y)}$ along $x(y)$ direction and on the dimensions of the simulation cell itself $\left(L_{x} a\right) \times\left(L_{y} a\right)$, where $a$ is the mesh unit size. In order to obtain equilibrium configurations we start from a suitably chosen initial configuration and let the system evolve according to a purely relaxational dynamics appropriate for systems with a nonconserved order parameter $^{31}$

$$
\frac{\partial \mathbf{m}(\mathbf{r})}{\partial t}=-\gamma \frac{\delta F}{\delta \mathbf{m}(\mathbf{r})},
$$

where $\gamma$ is a dissipation coefficient.

\section{SIMULATION RESULTS}

In this section we present the results obtained by numerical simulations of the continuum model described in the previous section. For all the results presented hereafter, the model parameters have been set to $G=0.1, \varepsilon=12.5, \sigma=100$, $a=6.25$, and $\gamma=1$. With these model parameters, the Curie temperature in the absence of disorder ${ }^{30}$ is $T_{c}=1.157$. Thermodynamic quantities have been obtained on a system size of $L_{x}=L_{y}=256$ with $n_{x}=8$ and $n_{y}=64$ whereas magnetic tweed patterns are presented on a larger system size of $L_{x}$ $=L_{y}=512$ and $n_{x}=4, n_{y}=32$.

In Fig. 1 we show the configurations at different temperatures for the simulation variable $m_{y}$ at each site obtained by relaxation of an initial homogeneously magnetized sample. The gray scale intensity at each snapshot is normalized according to the maximum value of the magnetization at the corresponding temperature. Therefore, white and black are up and down magnetized regions, respectively, whereas gray is indicative of a weakly magnetized region.

At low temperatures (e.g., $T=1.14$ ) the stable configuration exhibits the characteristic striped dipolar structure with net magnetization close to zero. It is also evident that around $T=1.155$ a transition occurs to an inhomogeneous structure where short wavelength magnetic modulations (magnetic tweed) coexist with nonmagnetized regions. The extent of the nonmagnetized regions increases with temperature and the extent of the magnetic tweed reduces asymptotically. When the amount of magnetic tweed is sufficiently small, the behavior of the system is expected to be purely paramagnetic.

In order to characterize the different magnetic phases and transitions we study the temperature dependence of the local magnetization distribution and its associated domain size distribution, the inverse magnetic susceptibility and the magnetized volume fraction. From the behavior of this last quantity we expect to elucidate whether or not the onset of tweed in the paramagnetic phase can be related to a phase transition.

\section{A. Local magnetization distribution}

From Fig. 1 it is clear that at low temperature the equilibrium configurations are of dipolar type. We thus start by obtaining the relaxed configurations at the lowest temperature studied, $T=1.13$. This is achieved by relaxation of suitably generated dipolar structures with a characteristic length as close as possible to the one that minimizes the free energy. This procedure avoids the pinning of the system to higher energy metastable states. We note that the magnetic configurations always relax to metastable states and we never obtain the true equilibrium configurations. At higher temperatures the relevant configurations are then obtained by heating the relaxed configurations corresponding to $T=1.13$. At each temperature we study the local magnetization distribution and perform averages over 75 independent realizations. The results are shown in Fig. 2.

The dipolar and paramagnetic phases are clearly identified by a double and a single peak in the local magnetization distribution, respectively. The existence of magnetic tweed in the paramagnetic phase (above the Curie temperature $T_{c}$ $\sim 1.155)$ is reflected in the width of the peak of the magnetization density at $m=0$, which reveals that at zero applied field there exist magnetized regions. The Curie temperature cannot be unambiguously obtained from this data alone, since there is a temperature range where three peaks are observed.

\section{B. Inverse magnetic susceptibility}

In tweed studies, the knowledge of the relevant response function is of crucial importance for both theoretical 


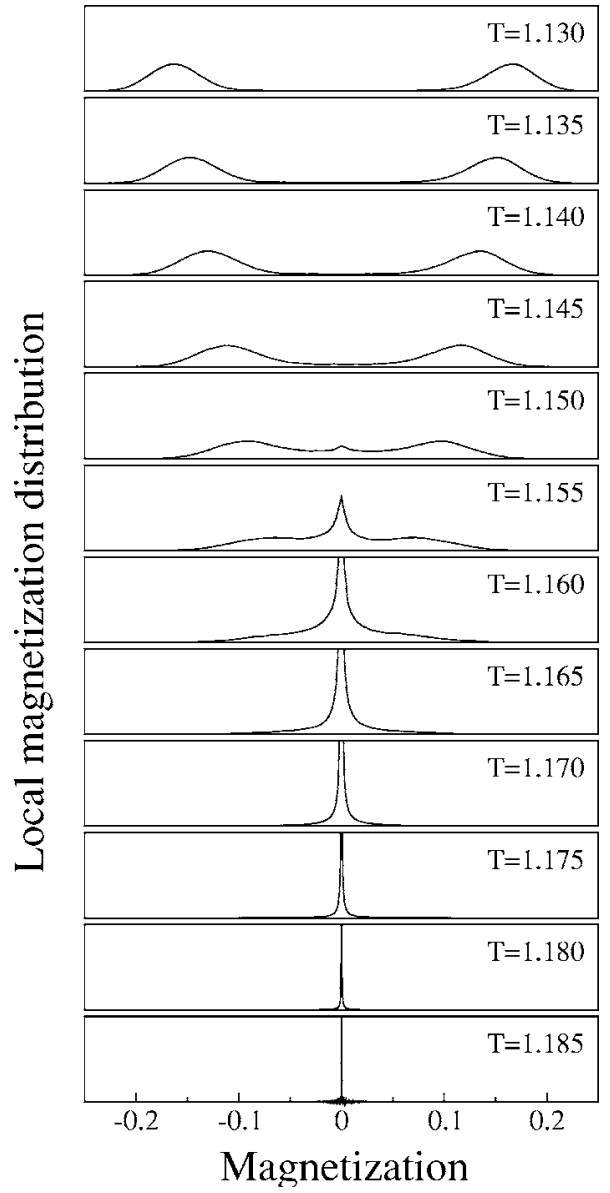

FIG. 2. Local magnetization distribution at different temperatures for a system size defined by $L_{x}=L_{y}=256, n_{x}=8$, and $n_{y}=64$.

purposes and experiments. In Fig. 3 we depict the temperature behavior of the inverse magnetic susceptibility for individual disorder realizations. A cusp appears at the putative tweed onset temperature in the paramagnetic phase. How-

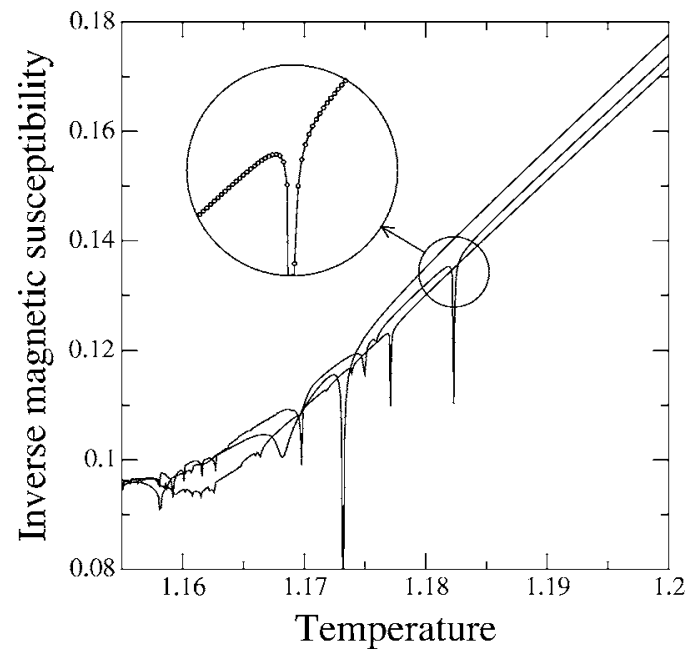

FIG. 3. Inverse magnetic susceptibility vs temperature for three individual disorder realizations. The inset shows the detailed structure of one of the peaks at the onset of magnetic tweed.

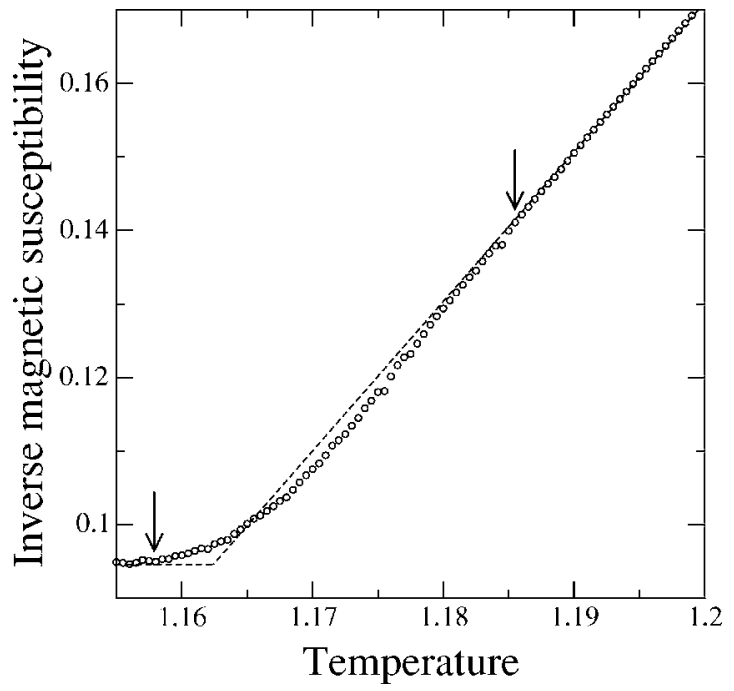

FIG. 4. Averaged inverse magnetic susceptibility vs temperature. Dashed lines indicate the expected behavior for the dipolar and paramagnetic phases. Arrows delimit the region of tweed.

ever, since the cusp temperature is different from one realization to another, it does not appear when the average over different configurations of disorder is performed. This is shown in Fig. 4 after averaging over 20 independent realizations of disorder. Apparently, there is no signature of a phase transition between the magnetic tweed and the paramagnetic phases. In any case, due to finite size effects and long-range interactions, ${ }^{32}$ the behavior of the magnetic susceptibility of a very large system may be different from that shown in Fig. 4. The stability region of magnetic tweed (indicated by arrows) is identified as the region that deviates from the linear Curie behavior in the paramagnetic phase. Furthermore, this is consistent with the behavior of the local magnetization distribution (Fig. 2). Analogous to previous suggestions formulated in the context of structural tweed, ${ }^{8}$ we find that the signature of precursor magnetic modulations in the response function is an enhanced sensitivity of the material to an external magnetic field.

\section{Magnetized volume fraction and phase diagram}

We start by defining a pseudo-order parameter suitable for the characterization of the transition between the different phases in the presence of disorder. Since at high enough temperature (in the purely paramagnetic phase) the local magnetization vanishes and at low temperature (in the dipolar phase) it is nonzero everywhere in the system except at the magnetic interfaces, we might define the fraction of magnetized volume (or area in 2D) of the simulation cell as the "order parameter" for both the paramagnetic-tweed and tweed-dipolar transitions. This is shown in Fig. 5 for different values of the strength of disorder. In the limit of zero disorder, the temperature dependence of the pseudo-order parameter would be a steplike function at the Curie temperature since the nominal transition temperature is the same everywhere in the system. In the presence of disorder, the existence of a temperature range where magnetized and nonmag- 


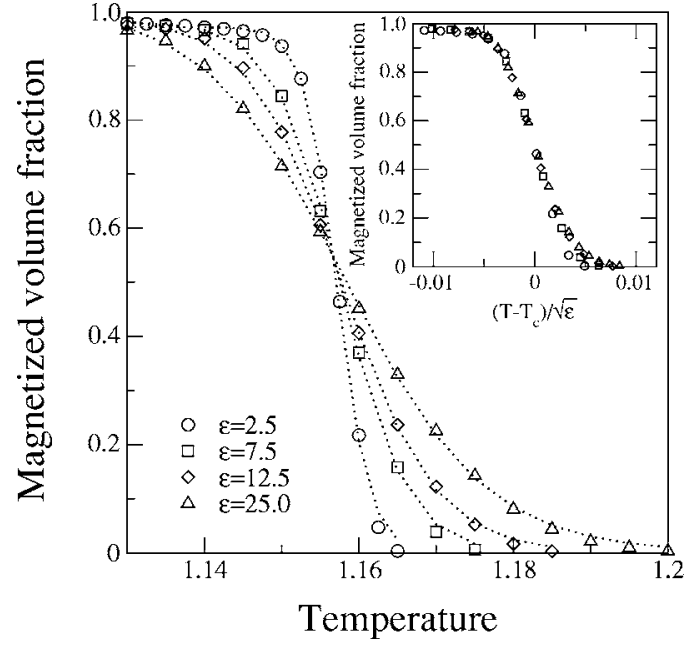

FIG. 5. Temperature behavior of the magnetized volume fraction. The pseudo-order parameter for different values of the strength of disorder. $L_{x}=L_{y}=256, n_{x}=8$, and $n_{y}=64$. The inset shows the scaling of different curves.

netized regions coexist, smoothes out the temperature dependence of the order parameter. Moreover, it is clear that as the strength of disorder increases the temperature range of such an intermediate tweed region with coexisting magnetized and nonmagnetized regions also increases.

The Curie temperature is identified as the inflection point of the curve and it is nearly independent of the strength of disorder. The stability range of the magnetic tweed is a more subtle question. From Fig. 5 it is evident that the fraction of magnetized regions vanishes asymptotically as the temperature is raised. Thus, we establish that the stability range of magnetic tweed comprises the Curie temperature and the temperature at which the fraction of magnetized regions is $1 \%$ of its low-temperature value. We stress that this criterion yields a temperature range of stability for tweed consistent with that arising from the temperature behavior of both the

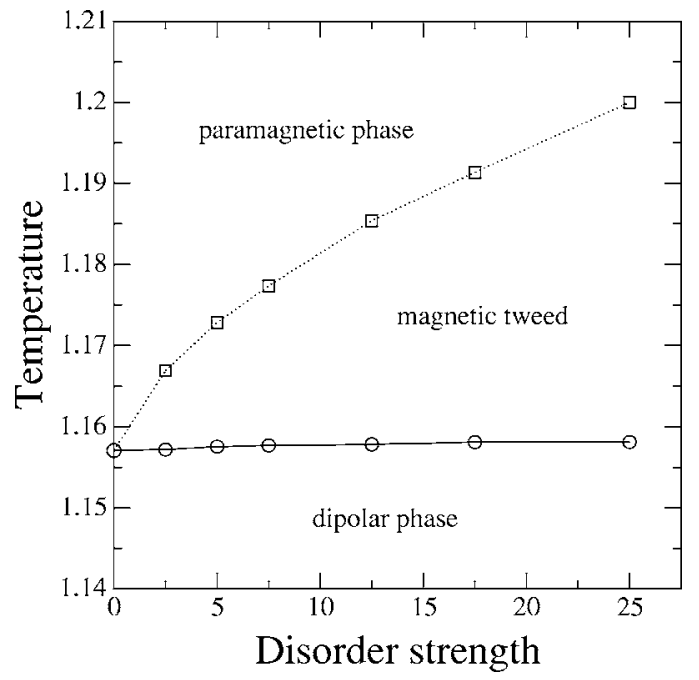

FIG. 6. Phase diagram: disorder strength vs temperature. Note that $T_{c}>1$ at zero disorder strength due to the effect of the dipolar interaction (see Ref. 30). local magnetization distribution and the inverse magnetic susceptibility presented above. Moreover, it scales as the square root of the disorder strength $\varepsilon$. In the inset of Fig. 5 we depict the collapse of different curves when the stability range of temperature is divided by the square root of the disorder strength.

Next, we use the information in Fig. 5 to construct the temperature-disorder strength phase diagram, shown in Fig. 6 . There is a well defined phase transition between the dipolar (ferromagnetic) and the magnetic tweed phases. From our present results it is difficult to conclude whether this transition is second order or weakly first order. In general, this may depend on the details of the model such as the crystal field or some properties of the disorder. Nonetheless, this transition has been reported experimentally to be of second order. ${ }^{15}$ The magnetic tweed is a paramagnetic textured phase and its range of stability increases with the disorder strength. From our results, it follows that the purely paramagnetic and the textured tweedlike paramagnetic phases are separated by a crossover.

\section{Size distribution of magnetic domains}

We end the thermodynamic characterization studies by computing the size distribution of magnetic domains corre-

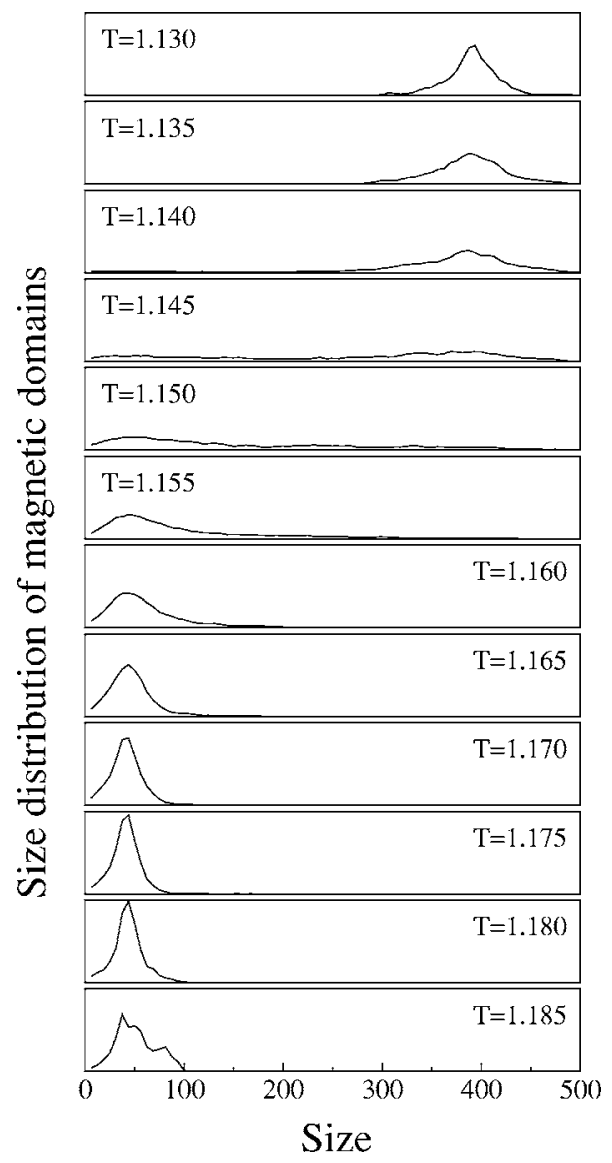

FIG. 7. Size distribution of magnetic domains in the $\hat{x}$ direction at different temperatures. 
sponding to the same configurations used to obtain the local magnetization distribution in Fig. 2. The temperature dependence of the size distribution along the direction perpendicular to the magnetization vector is shown in Fig. 7. Interestingly, the two modulated structures, that is the dipolar and the magnetic tweed, appear clearly differentiated.

The low-temperature peak is related to the modulation of the dipolar phase. As we already mentioned, this modulation length scales as the square root of the system size. ${ }^{28}$ At higher temperatures, inside the stability regime of magnetic tweed, we also obtain a well defined single peak. Note that it abruptly switches to bigger sizes when crossing the Curie point. The tweed modulation length scale should arise from either surface effects or the disorder correlation length $\sigma$ or a combination thereof. We have performed some preliminary simulations that seem to indicate that the tweed length is related to the disorder correlation length. Nevertheless, large scale computations are required to confirm this important point.

\section{CONCLUDING REMARKS}

Magnetic tweed can be defined as real space TEM (or other) observations corresponding to modulations in the magnetization above the Curie temperature. We have demonstrated the presence of stripelike magnetic tweed within a Ginzburg-Landau (purely) magnetic model with quenched-in disorder. Notice that since we work at the lifetime scale of disorder, which is much larger than the time scale of magnetic relaxation, the tweed modulations thus obtained will be static (or long lived). The model is then suitable to describe a magnetic alloy with intrinsic statistical compositional fluctuations as the source of static disorder, leading to a local transition temperature that spatially fluctuates from one point to another. The existence of regions where the local transition temperature is larger than the average bulk (Curie) transition will produce pretransitional magnetic domains in an essentially paramagnetic system. The stripelike correlations is then the global response to nonlocal anisotropic longrange interactions between such domains.

In this work we have devised different ways of characterizing magnetic tweed in terms of local magnetization distribution and inverse magnetic susceptibility. The latter quantity can be measured experimentally to test our prediction of its nonlinear behavior above the Curie temperature. Whereas anomalies in response functions have been identified as being connected with the presence of tweed modulations, ${ }^{8,23}$ here we have provided evidence that in turn the precursor modulations enhance the sensitivity to external fields. Moreover, we expect anomalies in the magnon dispersion due to the magnetic tweed at temperatures $T>T_{c}$ (akin to phonon anomalies above the structural transition temperature in the case of structural tweed ${ }^{7}$ ).

A similar model for understanding ferroelectric or polar tweed $^{12-14}$ can be used with magnetization (axial) vector $\mathbf{m}$ replaced by the polarization vector $\mathbf{P}$. Note that the form of the electric dipolar interaction is the same as that for the magnetic dipolar interaction. However, unlike free charges, there are no isolated magnetic charges (monopoles) and thus the surface effects and crystalline anisotropy effects would be different in these two cases. Note that the model for the magnetic tweed considered here, or a generalized model for a ferroic tweed, satisfies the three generic criteria for the existence of precursor modulations: a sensitivity of the magnetic or relevant order parameter to local coupling to disorder, the presence of (dipolar) long-range forces to produce a global response from local perturbations, and anisotropy that selects modulations along specific directions. Note also that the long-range force arising from elastic compatibility in the case of structural tweed ${ }^{8,9}$ can be expressed as an elastic dipolar interaction. ${ }^{23}$

A logical continuation of the present research is to extend the mode ${ }^{23}$ and simulations to the magnetoelastic tweed, which is observed below the Curie point but above the structural transition temperature. Nonetheless, it can be simulated using a similar model with either the magnetic and/or structural disorder present and a coupling of strain with magnetization. ${ }^{23}$ Magnetoelastic tweed patterns are crosshatched magnetic modulations and always coexist with similar strain modulations. This is a consequence of a strong magnetoelastic coupling (and the multiferroic character of these materials) as evidenced by the experimental observations in the Heusler alloy ${ }^{33} \mathrm{Ni}_{2} \mathrm{MnGa}$. Other alloys that exhibit magnetoelastic tweed include ${ }^{16,18} \quad \mathrm{Co}_{2} \mathrm{NiGa}$ and ${ }^{19}$ $\mathrm{Ni}_{2} \mathrm{FeGa}$. Thermodynamic characterization of tweed in these alloys and the utilization of their nanoscale magnetic modulations in specific applications remain an experimental challenge.

\section{ACKNOWLEDGMENTS}

This work was supported by CICyT (Spain) Project No. MAT-2004-1291, Marie-Curie RTN MULTIMAT (Contract No. MRTN-CT-2004-505226), CIRIT (Catalonia) Project No. 2001SGR00066, and the U.S. Department of Energy.

\section{APPENDIX: DIPOLAR INTERACTION}

In this appendix we give details of the computation of the dipolar interaction in a finite system. In an infinite system the magnetic dipolar interaction in two dimensions in Fourier space is given by Eq. (6), that can be readily obtained by substitution of the magnetization in Eq. (5) with its expression in terms of its Fourier transform

$$
\mathbf{m}(\mathbf{r})=\int d \mathbf{k} \exp (-i \mathbf{k} \cdot \mathbf{r}) \mathbf{m}(\mathbf{k})
$$

and solving the following integrals:

$$
\begin{gathered}
I_{y^{2}-x^{2}}(\mathbf{k})=\int d \mathbf{r} \exp (-i \mathbf{k} \cdot \mathbf{r}) \frac{y^{2}-x^{2}}{r^{4}}=\pi \frac{k_{x}^{2}-k_{y}^{2}}{k^{2}}, \\
I_{x y}(\mathbf{k})=\int d \mathbf{r} \exp (-i \mathbf{k} \cdot \mathbf{r}) \frac{x y}{r^{4}}=-\pi \frac{k_{x} k_{y}}{k^{2}} .
\end{gathered}
$$

In order to obtain a similar expression for a finite system we assume that the whole system is a finite number of copies of the simulated cell. In such a case, Eq. (5) reads 


$$
\begin{aligned}
F_{\text {dip }}= & \frac{1}{2} G a^{2} \sum_{x_{1}=1}^{n_{x} L_{x}} \sum_{y_{1}=1}^{n_{y} L_{y}} \sum_{x_{2}=1}^{n_{x} L_{x}} \sum_{y_{2}=1}^{n_{y} L_{y}}\left\{\frac{\left(y_{2}-y_{1}\right)^{2}-\left(x_{2}-x_{1}\right)^{2}}{\left[\left(x_{2}-x_{1}\right)^{2}+\left(y_{2}-y_{1}\right)^{2}\right]^{2}}\left[m_{x}\left(\mathbf{r}_{1}\right) m_{x}\left(\mathbf{r}_{2}\right)-m_{y}\left(\mathbf{r}_{1}\right) m_{y}\left(\mathbf{r}_{2}\right)\right]\right. \\
& \left.-2 \frac{\left(x_{2}-x_{1}\right)\left(y_{2}-y_{1}\right)}{\left[\left(x_{2}-x_{1}\right)^{2}+\left(y_{2}-y_{1}\right)^{2}\right]^{2}}\left[m_{x}\left(\mathbf{r}_{1}\right) m_{y}\left(\mathbf{r}_{2}\right)+m_{y}\left(\mathbf{r}_{1}\right) m_{x}\left(\mathbf{r}_{2}\right)\right]\right\},
\end{aligned}
$$

where $a$ is the discretization parameter, $L_{x}\left(L_{y}\right)$ is the number of points in which the $x(y)$ coordinate of the simulated cell is discretized, and $n_{x}\left(n_{y}\right)$ is the number of copies of the simulated cell in the $x(y)$ direction.

Even though the magnetization $\mathbf{m}(\mathbf{r})$ is no longer a periodic function, we can still define $\mathbf{m}(\mathbf{k})$ as the Fourier transform of the periodic repetition of the values of the magnetization within the simulation cell. Therefore, within this cell the magnetization can be written as

$$
\mathbf{m}(\mathbf{r})=\sum_{\xi_{x}=0}^{L_{x}-1} \sum_{\xi_{y}=0}^{L_{y}-1} \exp (-i \mathbf{k} \cdot \mathbf{r}) \mathbf{m}(\mathbf{k})
$$

where $\mathbf{k}=(2 \pi / a)\left(\xi_{x} / L_{x}, \xi_{y} / L_{y}\right)$. We introduce this expression into Eq. (A3), and after some algebra we obtain

$$
\begin{aligned}
F_{\text {dip }}= & \frac{1}{2} G a^{2} n_{x} L_{x} n_{y} L_{y} \sum_{\xi_{x}=0}^{L_{x}-1} \sum_{\xi_{y}=0}^{L_{y}-1} \sum_{\xi_{x}^{\prime}=0}^{L_{x}-1} \sum_{\xi_{y}^{\prime}=0}^{L_{y}-1} \\
& \times\left\{\left[m_{x}\left(\mathbf{k}^{\prime}\right) m_{x}(\mathbf{k})-m_{y}\left(\mathbf{k}^{\prime}\right) m_{y}(\mathbf{k})\right] S_{y^{2}-x^{2}}\left(-\mathbf{k}-\mathbf{k}^{\prime}, \mathbf{k}\right)\right. \\
& \left.-2\left[m_{x}\left(\mathbf{k}^{\prime}\right) m_{y}(\mathbf{k})+m_{y}\left(\mathbf{k}^{\prime}\right) m_{x}(\mathbf{k})\right] S_{x y}\left(-\mathbf{k}-\mathbf{k}^{\prime}, \mathbf{k}\right)\right\},
\end{aligned}
$$

where

$$
\begin{aligned}
S_{y^{2}-x^{2}}\left(\mathbf{k}^{\prime \prime}, \mathbf{k}\right)= & \frac{1}{L_{x} L_{y}} \sum_{x=1}^{L_{x}} \sum_{y=1}^{L_{y}} \exp \left(i \mathbf{k}^{\prime \prime} \cdot \mathbf{r}\right) \sum_{x^{\prime}=1-x}^{L_{x}-x} \sum_{y^{\prime}=1-y}^{L_{y}-y} \\
& \times \exp \left(-i \mathbf{k} \cdot \mathbf{r}^{\prime}\right) s_{y^{2}-x^{2}}\left(\mathbf{r}^{\prime}\right), \\
S_{x y}\left(\mathbf{k}^{\prime \prime}, \mathbf{k}\right)= & \frac{1}{L_{x} L_{y}} \sum_{x=1}^{L_{x}} \sum_{y=1}^{L_{y}} \exp \left(i \mathbf{k}^{\prime \prime} \cdot \mathbf{r}\right) \sum_{x^{\prime}=1-x}^{L_{x}-x} \sum_{y^{\prime}=1-y}^{L_{y}-y} \\
& \times \exp \left(-i \mathbf{k} \cdot \mathbf{r}^{\prime}\right) s_{x y}\left(\mathbf{r}^{\prime}\right)
\end{aligned}
$$

and

$$
\begin{aligned}
s_{y^{2}-x^{2}}(\mathbf{r})= & \frac{1}{n_{x} n_{y}} \sum_{\alpha=-n_{x}+1}^{n_{x}-1}\left(n_{x}-|\alpha|\right) \sum_{\beta=-n_{y}+1}^{n_{y}-1} \\
& \times\left(n_{y}-|\beta|\right) \frac{\left(y+\beta L_{y}\right)^{2}-\left(x+\alpha L_{x}\right)^{2}}{\left[\left(x+\alpha L_{x}\right)^{2}+\left(y+\beta L_{y}\right)^{2}\right]^{2}},
\end{aligned}
$$

$$
\begin{aligned}
s_{x y}(\mathbf{r})= & \frac{1}{n_{x} n_{y}} \sum_{\alpha=-n_{x}+1}^{n_{x}-1}\left(n_{x}-|\alpha|\right) \sum_{\beta=-n_{y}+1}^{n_{y}-1} \\
& \times\left(n_{y}-|\beta|\right) \frac{\left(x+\alpha L_{x}\right)\left(y+\beta L_{y}\right)}{\left[\left(x+\alpha L_{x}\right)^{2}+\left(y+\beta L_{y}\right)^{2}\right]^{2}} .
\end{aligned}
$$

Notice that since the system is finite the dipolar free energy in Fourier space is not local. Nevertheless, if the whole system is much larger than the simulation cell $\left(n_{x} \gg 1, n_{y}\right.$ $\gg 1)$ then the functions $s_{y^{2}-x^{2}}, s_{x y}$ are quasiperiodic with the periodicity of the simulation cell size. In such a case, the functions $S_{y^{2}-x^{2}}\left(\mathbf{k}^{\prime \prime}, \mathbf{k}\right), S_{x y}\left(\mathbf{k}^{\prime \prime}, \mathbf{k}\right)$ are small for $\mathbf{k}^{\prime \prime} \neq 0$ and, thus, we take into account only the term $\mathbf{k}^{\prime}=-\mathbf{k}$ in Eq. (A5). This leads to the following expression for the dipolar free energy in Fourier space:

$$
\begin{aligned}
F_{\text {dip }} \approx & \frac{1}{2} G n_{x} L_{x} n_{y} L_{y} \sum_{\xi_{x}=0}^{L_{x}-1} \sum_{\xi_{y}=0}^{L_{y}-1}\left\{\left[m_{x}(-\mathbf{k}) m_{x}(\mathbf{k})\right.\right. \\
& \left.-m_{y}(-\mathbf{k}) m_{y}(\mathbf{k})\right] S_{y^{2}-x^{2}}(0, \mathbf{k})-2\left[m_{x}(-\mathbf{k}) m_{y}(\mathbf{k})\right. \\
& \left.\left.+m_{y}(-\mathbf{k}) m_{x}(\mathbf{k})\right] S_{x y}(0, \mathbf{k})\right\},
\end{aligned}
$$

where the functions $S_{y^{2}-x^{2}}(0, \mathbf{k}), S_{x y}(0, \mathbf{k})$ can be rewritten as

$$
\begin{aligned}
S_{y^{2}-x^{2}}(0, \mathbf{k})= & \frac{1}{L_{x} L_{y}} \sum_{x=-L_{x}+1}^{L_{x}-1}\left(L_{x}-|x|\right) \sum_{y=-L_{y}+1}^{L_{y}-1} \\
& \times\left(L_{y}-|y|\right) \exp (i \mathbf{k} \cdot \mathbf{r}) S_{y^{2}-x^{2}}(\mathbf{r}),
\end{aligned}
$$

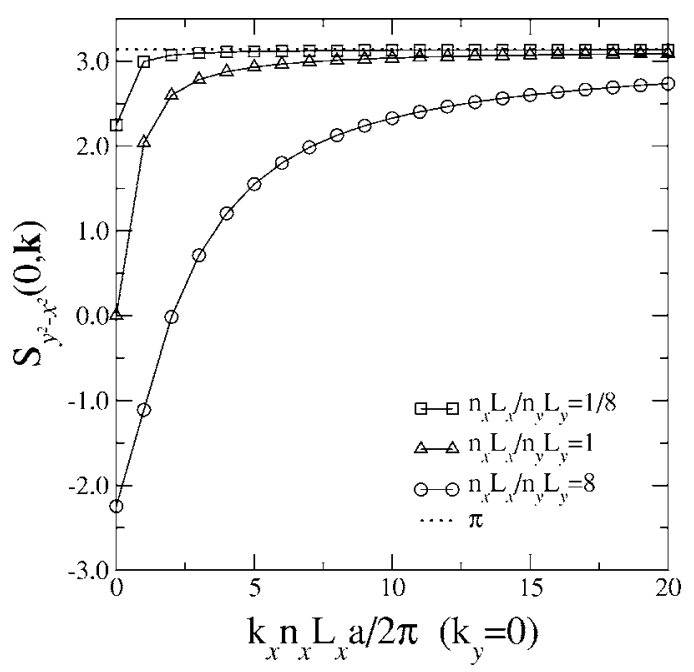

FIG. 8. Scaled function $S_{y^{2}-x^{2}}(0, \mathbf{k})$ for different degrees of shape anisotropy. 


$$
\begin{aligned}
S_{x y}(0, \mathbf{k})= & \frac{1}{L_{x} L_{y}} \sum_{x=-L_{x}+1}^{L_{x}-1}\left(L_{x}-|x|\right) \sum_{y=-L_{y}+1}^{L_{y}-1} \\
& \times\left(L_{y}-|y|\right) \exp (i \mathbf{k} \cdot \mathbf{r}) s_{x y}(\mathbf{r}) .
\end{aligned}
$$

Thus, in a finite system the integrals $I_{y^{2}-x^{2}}(\mathbf{k}), I_{x y}(\mathbf{k})$ defined in Eq. (A2) are replaced by the functions $S_{y^{2}-x^{2}}(0, \mathbf{k})$, $S_{x y}(0, \mathbf{k})$.

In Fig. 8 we plot the function $S_{y^{2}-x^{2}}(0, \mathbf{k})$ vs the scaled inverse wavelength $n_{x} L_{x} a / \lambda$ for $\mathbf{k}=(k, 0)$. This function scales with the system size $n_{x} L_{x} a$, as $S_{y^{2}-x^{2}}[0, \mathbf{k}=(k, 0)]$ $=\widetilde{S}\left(k n_{x} L_{x} a\right)$. The relevant result is that this function depends on the wavelength $\lambda$, the dipolar energy being lower for smaller $\lambda$. Therefore, in finite systems the competition between the dipolar interaction and the gradient term of the Ginzburg-Landau free energy stabilizes a given wavelength for the magnetic structure. Moreover, in Fig. 8 we also show that the function $S_{y^{2}-x^{2}}[0, \mathbf{k}=(k, 0)]$ depends on shape anisotropy, in particular its value at $\mathbf{k}=0$, which is related to the energy cost of a net magnetization. Similar statements can be made about the behavior of $S_{x y}$. However, since we are only interested here in the magnetization pointing in the [10] direction (and not in the [11] direction) we do not elaborate on this point any further.
${ }^{1}$ E. K. H. Salje, Phase Transformations in Ferroelastic and Coelastic Crystals (Cambridge University Press, Cambridge, UK, 1990).

${ }^{2}$ Shape Memory Materials, edited by K. Otsuka and C. M. Wayman (Cambridge University Press, Cambridge, UK, 1998).

${ }^{3}$ L. E. Tanner, A. R. Pelton, and R. Gronsky, J. Phys. (Paris) 12, C4-169 (1982).

${ }^{4}$ For a review see T. Castán, E. Vives, Ll. Mañosa, A. Planes, and A. Saxena, Magnetism and Structure in Functional Materials, edited by A. Planes, Ll. Mañosa, and A. Saxena (Springer, Berlin, 2005).

${ }^{5}$ S. M. Shapiro, J. Z. Larese, Y. Noda, S. C. Moss, and L. E. Tanner, Phys. Rev. Lett. 57, 3199 (1986).

${ }^{6}$ M. Liu, T. R. Finlayson, T. F. Smith, and L. E. Tanner, Mater. Sci. Eng., A 157, 225 (1992).

${ }^{7}$ J. A. Krumhansl, Nonlinearity in Condensed Matter, edited by A. R. Bishop, D. K. Campbell, P. Kumar, and S. E. Trullinger (Springer-Verlag, Berlin, 1987), p. 255.

${ }^{8}$ S. Kartha, J. A. Krumhansl, J. P. Sethna, and L. K. Wickham, Phys. Rev. B 52, 803 (1995).

${ }^{9}$ S. R. Shenoy, T. Lookman, A. Saxena, and A. R. Bishop, Phys. Rev. B 60, R12 537 (1999).

${ }^{10}$ A. M. Bratkovsky, S. C. Marais, V. Heine, and E. K. H. Salje, J. Phys.: Condens. Matter 6, 3678 (1994).

${ }^{11}$ In alloys, the most natural source of such perturbations is statistical compositional fluctuations that give rise to static or long lived tweed.

${ }^{12}$ X. Meng, K. Z. Baba-Kishi, G. K. H. Pang, C. L. Choy, and H. S. Luo, Philos. Mag. Lett. 84, 191 (2004).

${ }^{13}$ O. Tikhomirov, H. Jiang, and J. Levy, Phys. Rev. Lett. 89, 147601 (2002).

${ }^{14}$ Z. Xu, M. C. Kim, J. F. Li, and D. Viehland, Philos. Mag. A 74, 395 (1996)

${ }^{15}$ Y. Murakami, D. Shindo, K. Oikawa, R. Kainuma, and K. Ishida, Acta Mater. 50, 2173 (2002).

${ }^{16}$ Y. Kishi, M. De Graef, C. Craciunescu, T. A. Lograsso, D. A. Neumann, and M. Wuttig, J. Phys. IV 112, 1021 (2003).

${ }^{17}$ M. De Graef, Y. Kishi, and M. Wuttig, J. Phys. IV 112, 993 (2003).
${ }^{18}$ H. S. Park, Y. Murakami, D. Shindo, V. A. Chernenko, and T. Kanomata, Appl. Phys. Lett. 93, 3752 (2003).

${ }^{19}$ Y. Murakami, D. Shindo, K. Oikawa, R. Kainuma, and K. Ishida, Appl. Phys. Lett. 85, 6170 (2004).

${ }^{20}$ A. J. Millis, Solid State Commun. 126, 3 (2003).

${ }^{21}$ D. Shindo and T. Oikawa, Analytical Electron Microscopy for Materials Science (Springer, Berlin, 2002).

${ }^{22}$ S. P. Venkateswaran and M. De Graef, in Magnetism and Structure in Functional Materials (Ref. 4).

${ }^{23}$ A. Saxena, T. Castán, A. Planes, M. Porta, Y. Kishi, T. A. Lograsso, D. Viehland, M. Wuttig, and M. De Graef, Phys. Rev. Lett. 92, 197203 (2004).

${ }^{24}$ A. R. Bishop, T. Lookman, A. Saxena, and S. R. Shenoy, Europhys. Lett. 63, 289 (2003).

${ }^{25}$ B. P. Stojković, Z. G. Yu, A. L. Chernyshev, A. R. Bishop, A. H. Castro Neto, and N. Grønbech-Jensen, Phys. Rev. B 62, 4353 (2000).

${ }^{26}$ This is the signature of an instability of the single ferromagnetic domain state with respect to the formation of symmetry related magnetic domains.

${ }^{27}$ When considering the gradient contribution, its energy decreases as the wavelength of the magnetic modulation increases and the wavelength that minimizes the total free energy in this case is the maximum allowed by the simulation cell size.

${ }^{28}$ This well known result can be obtained from simple thermodynamic arguments. Moreover, we have verified that it is indeed reproduced in our system.

${ }^{29}$ The simplification concerning uniaxial anisotropy hinders the formation of topological defects such as vortices. Although the vortices are important in general, they have not been observed experimentally in the case of magnetic modulations in Heusler alloys above the Curie point.

${ }^{30}$ Notice that $T_{c}^{o}$ is the value for $G=0$. In general $T_{c}(G)=T_{c}^{o}$ $+\pi G / 2 A$

${ }^{31}$ A. J. Bray, Adv. Phys. 43, 357 (1994).

${ }^{32}$ A. G. Khachaturyan, in The Theory of Structural Transformations in Solids (Wiley, New York, 1983).

${ }^{33}$ A. Planes, E. Obradó, A. Gonzàlez-Comas, and Ll. Mañosa, Phys. Rev. Lett. 79, 3926 (1997). 